REVIEW ARTICLE

\title{
Health Coaching and Motivational Interventions for Diabetes and Hypertension Care
}

\author{
Thomas TH Wan ${ }^{1 *}$, Waleed Kattan ${ }^{2}$ and Amanda Terry \\ ${ }^{1}$ College of Health and Public Affairs, University of Central Florida, USA \\ ${ }^{2}$ Department of Health Services and Hospitals Administration, College of Economics and Management, King \\ Abdulaziz University, Saudi Arabia \\ ${ }^{3}$ Creation Health Research Center, Florida Hospital, USA
}

*Corresponding author: Thomas TH Wan, Ph.D., MHS, Professor and Associate Dean for Research, College of Health and Public Affairs, University of Central Florida, Orlando, FL 32816-3680, USA, Tel: 407-823-3678

\begin{abstract}
A systematic review on diabetes care was centered in their modifying effects of coaching and motivational interventions on diabetes and hypertension care. Critical issues pertaining to the dose-response relationship between the intervention program and outcomes, the cost-effectiveness evaluation, and the lengths of observation were noted. A positive impact of health coaching ( $\mathrm{HC}$ ) and motivational intervension (MI) was noted in this review of randomized controlled studies. However, successful results are dependent on many factors such as the type of the program, the ethnicity or racial composition coupled with cultural sensitivity, the training of the healthcare providers who will execute the program, and the characteristics of the patients who are involved in the program. Specific recommendations were made for future clinical trial studies, using health coaching $(\mathrm{HC})$ and motivational interview (MI) approaches.
\end{abstract}

\section{Keywords}

Health coaching, Motivational interview, Cost-effectiveness evaluation issues, Diabetes care, Hypertension control

Health coaching $(\mathrm{HC})$ and motivational interviewing (MI) are synergistic, yet distinctive, approaches in health education that can be employed to enhance selfefficacy and patient centered care with chronic illnesses such as diabetes and hypertension [1]. These diseases often leave a person with low energy, decreased cognitive strength, and weak motivation to live a healthy lifestyle. Additionally, lifestyle behaviors are learned and if individuals were never taught essential skills for adhering healthy diet and exercise, they may falter when making decisions based on food and activities. With the assistance of $\mathrm{HC}$ and $\mathrm{MI}$, persons may find both the encouragement and education needed to change the maladaptive behaviors that led them to or exacerbated their current conditions.

Randomized control studies pertaining to $\mathrm{HC}$ and $\mathrm{MI}$ for chronic conditions of the past 10 years show that many studies were concerned with the effect of $\mathrm{MI}$ or $\mathrm{HC}$ on diabetes care while only a few published articles were focused on hypertensive care. MI intervention studies were relatively heterogeneous in their approaches while health coaching usually consisted of goal setting, knowledge acquisition, individualized care and frequent follow up. The research designs and clinical implementations varied among those studies. A majority of these studies measured the results of $\mathrm{MI}$ and $\mathrm{HC}$ through enhancing patients' adherence to medications, achieving lower HBA1C, improving dietary control behaviors, and weight loss. Furthermore, most of the $\mathrm{MI}$ and $\mathrm{HC}$ studies showed statistically significant positive results in changing patients' behaviors and improving their health outcomes. The annotated research summary of selected randomized trials is detailed in Appendix 1.

Findings show that multi-component interventions targeting emotional, social, or family processes had a greater impact than interventions that just target a direct behavioral process and more effective outcomes when more sessions are delivered using an individu- 
alized approach [2]. In addition, targeting adherence rather than glycemic outcomes typically demonstrates greater result [3]. Another interesting issue noted is that the impact of health coaching was more impactful in high-risk patients [4].

The complexity involved in managing type 2 diabetes is also documented since motivating patients to change in attitude, knowledge, and behavioral practice is very challenging [5]. This might be one reason that only a few studies showed a significant effect of applying $\mathrm{HC}$ or $\mathrm{MI}$ on the overall health conditions of the patients [6]. In order to facilitate preventive practice it is imperative to understand the causal mechanisms leading to better patient care outcomes [5]. Another reason could be related to the implementation and delivery since different studies employed different types of clinicians with widely differing professional backgrounds, different types and intensity of $\mathrm{Ml}$ training, disparate $\mathrm{MI}$ doses provided, and a variety of practice settings [7]. Therefore, to improve patient outcomes, not only must the behavior of patients be changed but also that of providers and practices [6].

There are few highly-related worthy issues were discussed in the literature and needed more thoroughly reviewed to draw any solid conclusions. The first is the length of intervention. A wide variety of lengths of $\mathrm{HC}$ or $\mathrm{Ml}$ were considered in different studies. When interpreting the effectiveness of the intervention program, many studies showed that longer $\mathrm{HC}$ exposure resulted in better health outcomes [8]. Although many studies considered $<6$ month intervention is short and $>6$ month interval is long [9]. However, appropriate lengths of intervention effectiveness for varying patient population need to be defined. Thus, the dose-response relationship between the intervention and patient care outcomes can be ascertained.

The second issue is related to cost-effectiveness of the program. There is a wide discrepancy about the costeffectiveness in implementing such a program because of the presence of a wide spectrum of factors that need to be considered related to direct costs, indirect costs, and social costs $[10,11]$. The third issue is the short-term versus long-term effects of the intervention programs. Many studies showed that the short-term effect is higher than the long-term one $[9,12]$. The effects of longerterm intervention interval has yet to be determined.

In summary, a positive impact of $\mathrm{MI}$ and $\mathrm{HC}$ was noted in this review of randomized controlled studies. However, successful results are dependent on many factors such as the type of the program, the ethnicity or racial composition coupled with cultural sensitivity, the training of the healthcare providers who will execute the program, and the characteristics of the patients who are involved in the program. Moreover, important issues needed to be considered before implementing such programs are the duration of intervention, cost- effectiveness of the program, and the diversity of the patient population selected.

Future research on diabetes care and hypertension control studies, using $\mathrm{MI}$ and $\mathrm{HC}$, should explore the following options in conducting an implementation science project:

1. Use both experimental and quasi-experimental study designs: Ideally, a randomized controlled study design is preferred. However, in a populationbased study, it is feasible and reasonable to employ a propensity score matching and analytical approach so that the experimental or intervention group is comparable to the comparison group. Thus, the integrity of the experimental results can be ensured.

2. Define the components or stages of $\mathrm{MI}$ and $\mathrm{HC}$ interventions: The adoption of innovation or new preventive practice behavior in disease management is similar to the technology adoption model (TAM). Both patient reported outcomes and clinical outcomes should be carefully captured in the data collection in varying stages of the disease management process. Most importantly, the program should pay great attention to personalized or individualized care management as $\mathrm{MI}$ and $\mathrm{HC}$ activities are performed by trained coaches and motivators.

3. Consider the use of health information technology (HIT) to facilitate the adherence and compile relevant process and outcome indicators during the study period. Currently, numerous HIT based and commercially implemented health education modalities are available for patients. For instance, the HealthyTutor.Com has a useful product for introducing appropriate knowledge, attitude, and preventive health practice for diabetes and hypertension [13]. If this product can also include motivating and coaching strategies to assist the patients or providers, it will certainly optimize the power of health educational interventions for effective care management of diabetes and hypertension.

\section{References}

1. Simmons LA, Wolever RQ (2013) Integrative health coaching and motivational interviewing: Synergistic approaches to behavior change in healthcare. Glob Adv Health Med 2: 28-35.

2. Christie D, Channon S (2014) The potential for motivational interviewing to improve outcomes in the management of diabetes and obesity in paediatric and adult populations: A clinical review. Diabetes Obes Metab 16: 381-387.

3. Powell PW, Hilliard ME, Anderson BJ (2014) Motivational interviewing to promote adherence behaviors in pediatric type 1 diabetes. Curr Diab Rep 14: 531.

4. Basak Cinar A, Schou L (2014) Health promotion for patients with diabetes: Health coaching or formal health education? Int Dent J 64: 20-28. 
5. Ravmarathe K, Wan TTH, Marathe S (2016) A systematic review on the KAP-O framework for diabetes education and research. Medical Research Archives 4: 1-21.

6. Hayes E, McCahon C, Panahi MR, Hamre T, Pohlman K (2008) Alliance not compliance: Coaching strategies to improve type 2 diabetes outcomes. J Am Acad Nurse Pract 20: $155-162$.

7. DiLillo V, West DS (2011) Incorporating motivational interviewing into counseling for lifestyle change among overweight individuals with Type 2 diabetes. Diabetes Spectrum 24: 80-84.

8. Sherifali D, Viscardi V, Bai JW, Ali RM (2016) Evaluating the effect of a diabetes health coach in individuals with Type 2 diabetes. Can J Diabetes 40: 84-94.

9. Song D, Xu TZ, Sun QH (2014) Effect of motivational interviewing on self-management in patients with type 2 diabetes mellitus: A meta-analysis. International Journal of Nursing Sciences 1: 291-297.

10. Egede LE, Hernandez-Tejada MA (2011) Type I diabetes: Motivational enhancement therapy delivered with CBT by nurse therapists to people with type I diabetes leads to lowering of $\mathrm{HbA1C}$ values. Evid Based Ment Health 14: 19.

11. Ismail K, Maissi E, Thomas S, Chalder T, Schmidt U, et al. (2010) A randomised controlled trial of cognitive behavior therapy and motivational interviewing for people with Type 1 diabetes mellitus with persistent sub-optimal glycaemic control: A Diabetes and Psychological Therapies (ADaPT) study. Health Technol Assess 14: 1-101.

12. Greaves CJ, Middlebrooke A, O'Loughlin L, Holland S, Piper J, et al. (2008) Motivational interviewing for modifying diabetes risk: A randomised controlled trial. Br J Gen Pract 58: 535-540.

13. http://healthytutor.com/ 